\title{
Acute coronary syndrome in octogenarians: association between percutaneous coronary intervention and long-term mortality
}

\author{
Salim Bary Barywani' \\ Shijun Li ${ }^{1,2}$ \\ Maria Lindh' \\ Josefin Ekelund' \\ Max Petzold ${ }^{3}$ \\ Per Albertsson ${ }^{4}$ \\ Lars H Lund ${ }^{5,6}$ \\ Michael LX Fu'
}

'Department of Molecular and Clinical Medicine, Sahlgrenska Academy, Sahlgrenska University Hospital/Östra Hospital, Gothenburg, Sweden; ${ }^{2}$ Department of Geriatrical Cardiology, PLA General Hospitals, Beijing, People's Republic of China; ${ }^{3}$ Centre for Applied Biostatistics, University of Gothenurg,

Gothenburg, ${ }^{4}$ Department of Cardiology, Sahlgrenska University Hospital, University of Gothenburg, Gothenburg, ${ }^{5}$ Department of Medicine, Karolinska Institute, ${ }^{6}$ Department of Cardiology, Karolinska University Hospital, Stockholm, Sweden
This article was published in the following Dove Press journal:

Clinical Interventions in Aging

28 September 2015

Number of times this article has been viewed

Aim: Evidence of improved survival after use of percutaneous coronary intervention (PCI) in elderly patients with acute coronary syndrome (ACS) is limited. We assessed the association between PCI and long-term mortality in octogenarians with ACS.

Methods and results: We followed 353 consecutive patients aged $\geq 80$ years hospitalized with ACS during 2006-2007. Among them, 182 were treated with PCI, whereas 171 were not. PCI-treated patients were younger and more often male, and had less stroke and dependency in activities of daily living, but there were no significant differences in occurrence of diabetes mellitus, chronic obstructive pulmonary disease, hypertension, and uncured malignancies between the two groups. The association between PCI and all-cause mortality was assessed in the overall cohort and a 1:1 matched cohort based on propensity score (PS). In overall cohort, 5 -year all-cause mortality was $46.2 \%$ and $89.5 \%$ in the PCI and non-PCI groups, respectively. Cox regression analysis in overall cohort by adjustment for ten baseline variables showed statistically significant association between PCI and reduced long-term mortality $(P<0.001$, hazard ratio $0.4,95 \%$ confidence interval $[\mathrm{CI}] 0.2-0.5)$. In propensity-matched cohort, 5 -year all-cause mortality was $54.9 \%$ and $83.1 \%$ in the PCI and non-PCI groups, respectively. Kaplan-Meier survival curves and log rank test showed significantly improved mean survival rates $(P=0.001)$ : 48 months (95\% CI 41-54) for PCI-treated patients versus 35 months (95\% CI 29-42) for non-PCI-treated patients. Furthermore, by performing Cox regression analysis, PCI was still associated with reduced long-term mortality $(P=0.029$, hazard ratio 0.5 , 95\% CI $0.3-0.9)$ after adjustment for PS and confounders: age, male sex, cognitive deterioration, uncured malignancies, left ventricular ejection fraction $\leq 45 \%$, estimated glomerular filtration rate $\leq 35 \mathrm{~mL} / \mathrm{min}$, ST-segment elevation myocardial infarction, mitral regurgitation, and medication at discharge with clopidogrel and statins.

Conclusion: In octogenarians with ACS, PCI was associated with improved survival from all-cause death over 5 years of follow-up.

Keywords: percutaneous coronary intervention, mortality, octogenarians, acute coronary syndrome

\section{Introduction}

Current guidelines regarding use of percutaneous coronary intervention (PCI) in acute coronary syndrome (ACS) are based on data derived from randomized clinical trials, which were mostly conducted in younger patients, ${ }^{1-3}$ although a substantial number of patients with ACS are aged $\geq 80$ years. The available data in patients with ACS aged $\geq 80$ years are of short term and are mainly registry derived. ${ }^{4-6} \mathrm{Mid}$ - and long-term data regarding PCI in octogenarians are not only very limited but also inconsistent. ${ }^{7-10}$ For instance, incidence rates of major adverse cardiac events, defined as the combined
Correspondence: Salim Bary Barywan Department of Molecular and Clinical Medicine, Sahlgrenska Academy, Sahlgrenska University Hospital/ Östra Hospital, Smörslottsgatan I, 41685 Gothenburg, Sweden

Tel +46 3I 3421000

Email salim.barywani@vgregion.se 
events of death, revascularization, and myocardial infarction (MI), among elderly patients have been found to be higher than younger patients in some studies but similar to younger patients in other studies. ${ }^{7-10}$ Moreover, available observational studies have demonstrated that older age is associated with both higher in-hospital mortality and frequent complications such as renal failure and bleeding after PCI. ${ }^{11,12}$

Because of the paucity and inconsistency of data on the use of PCI in octogenarians in daily clinical practice, it is difficult for physicians to make well-grounded decisions on the use of PCI in these patients. Elderly patients have been found to be less likely than younger ones to undergo PCI, despite that it is contrary to prevailing ethical principles when PCI is withheld from elderly patients solely because of their older age. Nevertheless, there are existing data suggesting that older age is associated with negative outcomes. ${ }^{13-15}$ Moreover, the elderly constitute a heterogeneous group. The term "elderly" is a broad term comprising the "young" old (65-74 years), the "older" old (75-84 years), and the "oldest" old ( $\geq 85$ years), ${ }^{16}$ with considerable individual variation in comorbidities and physical capabilities. Undoubtedly, it is essential to address whether PCI can improve survival in an octogenarian group with ACS irrespective of their age and comorbidities. So far, there were two trials studying an aggressive approach in comparison with a conservative strategy. In a randomized study of 313 patients $\geq 75$ years of age with non-ST-segment elevation ACS, an early aggressive approach (coronary angiography, and when indicated, revascularization within 72 hours) resulted in a significant reduction in the primary endpoint (the composite of death, MI, disabling stroke, and repeated hospital stay for cardiovascular causes or severe bleeding within 1 year) in patients with elevated troponin on admission but not in those with normal troponin. Because of this significant interaction of the treatment effect according to troponin status at baseline, the benefit of an early aggressive approach among elderly patients with non-ST-segment elevation ACS was not conclusive. ${ }^{17}$ Recently, in a multicenter study conducted at the American College of Cardiology (ACC) on March 16, 2015, Tegn et al (unpublished data, 2015) reported that 457 stable ACS patients $>80$ years old were randomized to an invasive $(n=229)$ or conservative strategy $(n=228)$. In this study, those who underwent an invasive strategy had a decrease in the primary composite endpoint (MI, need for urgent revascularization, stroke, and death) at a median follow-up of 18 months as compared with a conservative approach (not published yet). However, no prospective, randomized, controlled clinical trials are available to compare head to head between PCI-treated and non-PCI-treated patients with ACS aged $\geq 80$ years. Therefore, decision-making about performing PCI in this age group remains highly empirical. The aim of the present study was to assess the association between PCI and long-term mortality in octogenarians with ACS.

\section{Materials and methods Study cohort}

Three hundred and fifty-three patients aged $\geq 80$ years who were hospitalized due to ACS, during 2006-2007 at two university hospitals (Sahlgrenska and Östra) affiliated with the University of Gothenburg, were included consecutively, and studied from January 2 to May 30, 2012. Among them, 182 were treated with PCI, whereas 171 were not. Patients came directly from ambulance or were referred from cardiology and medical departments at both hospitals. Treatment with PCI or no treatment was based on a pure clinical decision made by responsible cardiologist. All PCIs were performed at a joint PCI center for both hospitals. The time period 2006-2007 was chosen to allow for a follow-up period of at least 5 years. Patients were identified from the hospital registry. In cases that two or more PCI procedures were performed in the same patient, only the first PCI the individual patient underwent at an age $\geq 80$ years was studied. Two exclusion criteria for the study were applied. Firstly, patients belonging to other hospitals but undergoing PCI at our center were excluded due to the lack of medical records. Secondly, since the objective of the study was to evaluate the prognosis after PCI in ACS, elective PCI procedures with the indication of stable angina pectoris were excluded. This is based on the fact that the current PCI indication in case of stable angina is to relieve symptoms rather than for prognostic benefit. The study protocol was approved by the Ethical Committee at the University of Gothenburg.

\section{Statistics}

The results are presented as percentages and the mean \pm standard deviation. In the case of continuous variables that visual inspection of their histograms, normal $Q-Q$ plots and box plots showed that the variables were approximately normally distributed. Statistical analysis was performed using Student's unpaired $t$-test. Mann-Whitney test was used for non-normally distributed continuous variables. For discrete variables, the chi-square test was used. A $P$-value $<0.05$ was regarded as statistically significant. The hazard ratios (HRs) from Cox survival analysis with confidence intervals (CIs) and $P$-values were presented. Propensity score (PS)matching analysis with 1:1 nearest neighbor matching was employed. Variables used in developing the PS are presented in Table 1. These variables were chosen based on clinical 


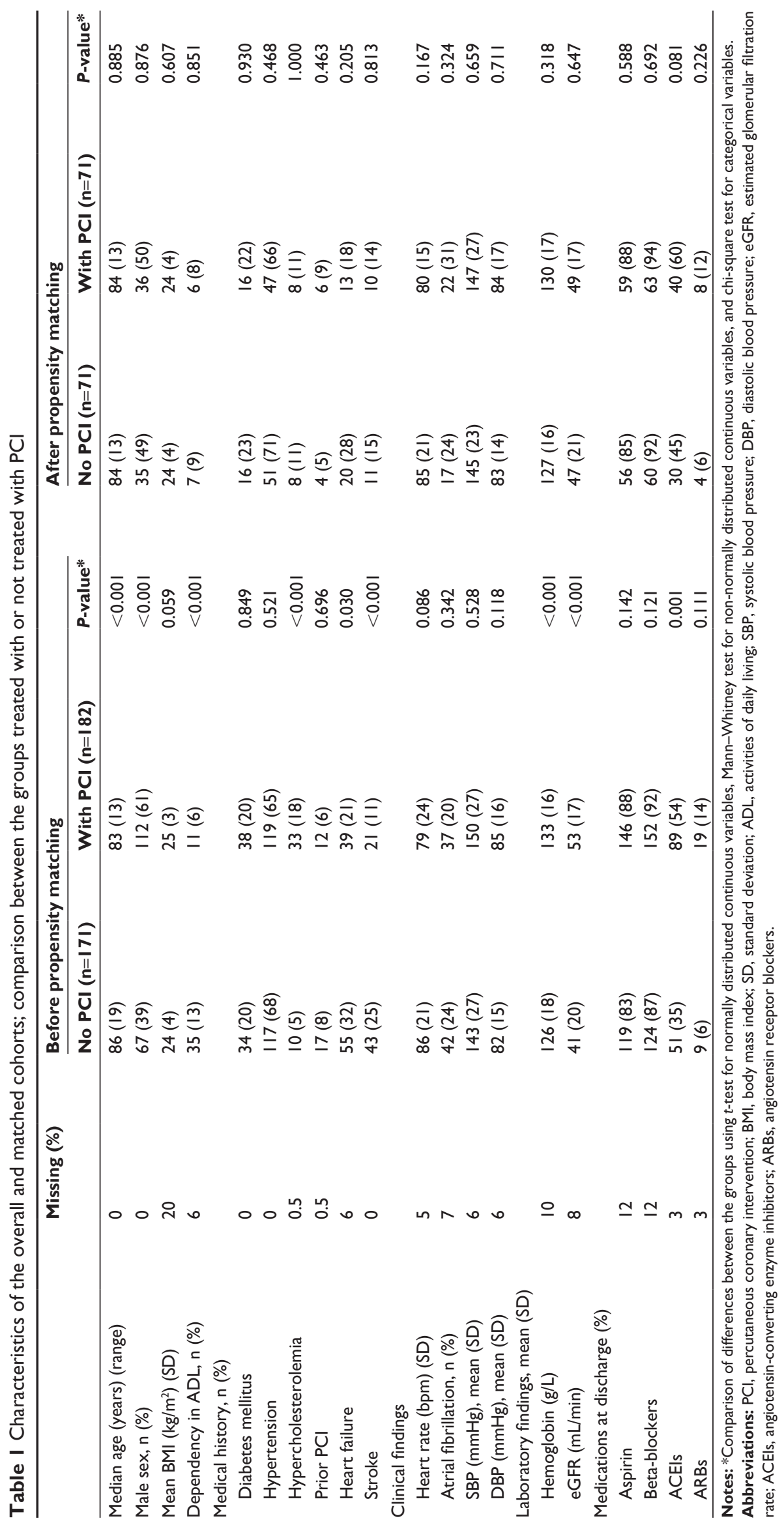


relevance. As shown in Figure 1, the covariate balance was improved in the matched sample, that the region of common support spanned almost the entire distribution of the PS, and that it was only in the extreme tail regions that no appropriate matching could be found. After PS matching, 142 patients remained including 71 in the PCI-treated and 71 in non-PCI-treated groups.

Both the overall cohort and the PS-matched cohort were further analyzed by Cox proportional-hazard regression models. Variables with clinical relevance and significant results from univariable Cox models were included in multivariable models (Table 2). Cox models were assessed for proportional-hazard assumption for covariates, graphically with Cox-adjusted log minus log curves and statically using Schoenfeld global test. Survival rates are presented both as adjusted Cox regression survival curves and as Kaplan-Meier curves with log rank test for comparisons.

\section{Clinical outcome data}

All clinical variables are based on medical records. The primary endpoint was all-cause mortality based on data from the death registry of the National Board of Health and Welfare in Sweden, which were available for all

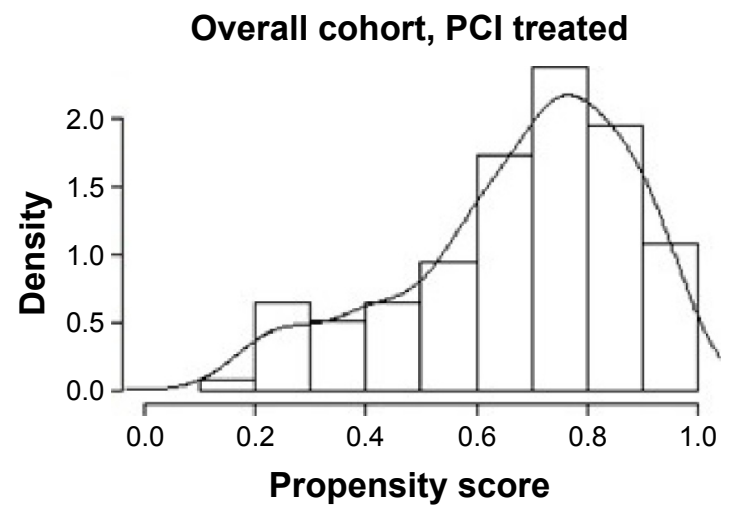

Overall cohort, non-PCI treated

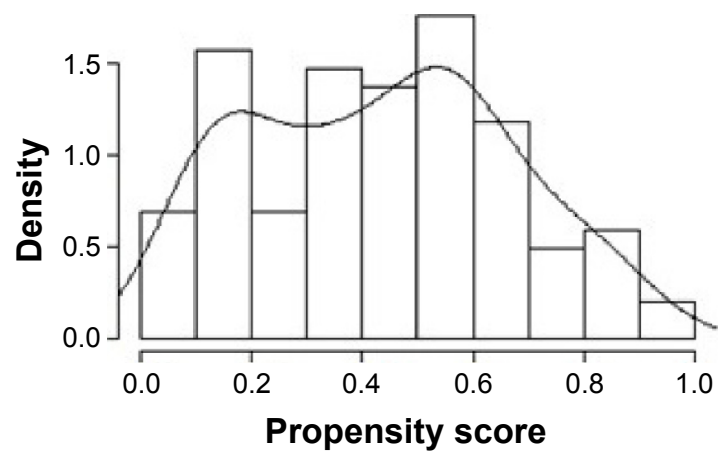

Figure I Distribution of propensity scores in the matched and overall cohort. Abbreviation: $\mathrm{PCl}$, percutaneous coronary intervention. studied patients during the time period from January 2 to May 30, 2012.

\section{Results}

\section{Clinical characteristics of the $\mathrm{PCl}$-treated and non- $\mathrm{PCl}$-treated group}

As shown in Table 1, in the overall cohort, the PCI-treated group was younger and more frequently male, and had less stroke and dependency in activities of daily living, higher left ventricular ejection fraction, and more often treatment with clopidogrel and statins compared with those in the non-PCItreated group. But no significant differences in occurrence of diabetes mellitus, chronic obstructive pulmonary disease, hypertension, and uncured malignancies were found between the two groups.

Indications for PCI were ST-segment elevation MI (STEMI; 52\%), non-ST-segment elevation MI (NSTEMI; $36 \%$, and unstable angina (11\%). In non-PCI group, $14 \%, 81 \%$, and $5 \%$ of patients had STEMI, NSTEMI, and unstable angina, respectively. Among PCI-treated patients, only two received drug-eluting stent, $51 \%$ had three-vessel coronary artery disease, and only $21 \%$ had one-vessel coronary artery disease. Moreover, 57\% received stent in left
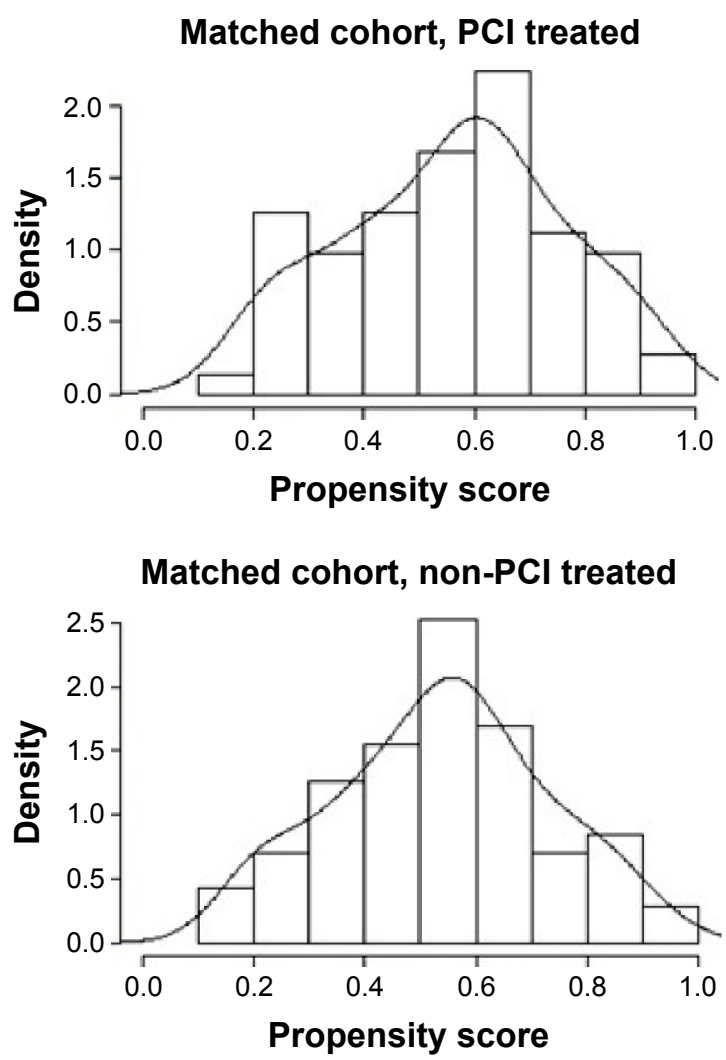
Table 2 Univariable ${ }^{+}$and multivariable Cox regression analyses for relationship between $\mathrm{PCl}$ and long-term all-cause mortality

\begin{tabular}{|c|c|c|c|c|}
\hline & \multicolumn{2}{|l|}{ Univariable } & \multicolumn{2}{|l|}{ Multivariable } \\
\hline & HR (95\% Cl) & $P$-value & HR (95\% Cl) & $P$-value \\
\hline \multicolumn{5}{|l|}{ In overall cohort } \\
\hline Unadjusted & $0.3(0.2-0.4)$ & $<0.00$ I & - & - \\
\hline Adjusted for the confounders* & - & - & $0.4(0.2-0.5)$ & $<0.001$ \\
\hline \multicolumn{5}{|l|}{ In matched cohort } \\
\hline Unadjusted & $0.5(0.3-0.7)$ & 0.002 & - & - \\
\hline Adjusted for PS & - & - & $0.6(0.2-1.9)$ & 0.003 \\
\hline Adjusted for PS and the confounders* & - & - & $0.5(0.2-0.9)$ & 0.020 \\
\hline
\end{tabular}

Notes: +Baseline variables including in univariable models: age, male sex, cognitive deterioration, diabetes mellitus, hypertension, uncured malignancies, left ventricular ejection fraction $\leq 45 \%$, estimated glomerular filtration rate $\leq 35 \mathrm{~mL} / \mathrm{min}$, ST-segment elevation myocardial infarction, atrial fibrillation, mitral regurgitation, chronic obstructive pulmonary disease, and medications at discharge with clopidogrel, beta-blockers, angiotensin-converting enzyme inhibitors/angiotensin receptor blockers, and statins. *Confounders including in multivariable models: age, male sex, cognitive deterioration, left ventricular ejection fraction $\leq 45 \%$, uncured malignancies, estimated glomerular filtration rate $\leq 35 \mathrm{~mL} / \mathrm{min}$, ST-segment elevation myocardial infarction, mitral regurgitation, and medications at discharge with clopidogrel and statins. Abbreviations: $\mathrm{PCl}$, percutaneous coronary intervention; $\mathrm{HR}$, hazard ratio; $\mathrm{Cl}$, confidence interval; PS, propensity score.

circumflex coronary artery or in left anterior descending coronary artery and only $4 \%$ in left main coronary artery. After PS matching, 142 patients remained including 71 in the PCI-treated and 71 in non-PCI-treated groups. As shown in Figure 1, the covariate balance was improved in the matched sample, that the region of common support spanned almost the entire distribution of the PS, and that it was only in the extreme tail regions that no appropriate matching could be found.

\section{All-cause 5-year mortality between the $\mathrm{PCl}$-treated and non-PCl-treated groups}

In the overall cohort ( $\mathrm{n}=353)$, all-cause mortality was $46.2 \%$ (84 events) and $89.5 \%$ (153 events) in those treated with PCI and those not treated with PCI, respectively. After PS matching ( $\mathrm{n}=142)$, all-cause mortality was $54.9 \%$ (39 events) and $83.1 \%$ (59 events) in the PCI and non-PCI subgroups, respectively. Kaplan-Meier survival curves and log rank test ( $P=0.001$ ) showed significantly improved survival in patients treated with PCI compared with patients not treated with PCI. The 5-year mean survival time after PCI was 48 months ( $95 \%$ CI 41-54), whereas 5-year mean survival time without PCI was 35 months (95\% CI 29-42) (Figure 2).

\section{Associations between $\mathrm{PCl}$ and long-term mortality}

A Cox proportional-hazard regression analysis was performed to assess association between PCI and long-term mortality, both in overall cohort and in propensity-matched cohort. In overall cohort $(n=353)$, Cox regression analysis showed that treatment with PCI was associated with reduced 5-year all-cause mortality (HR 0.4 with 95\% CI 0.3-0.6, $P<0.001$ ) after adjustment for ten baseline variables (Table 2 and Figure 3). Moreover, in propensity-matched group, $\mathrm{PCI}$ was still associated with reduced 5-year all-cause mortality (HR 0.5 with $95 \%$ CI $0.2-0.9, P=0.020$ ) after adjustment for PS and confounders including age, male sex, cognitive deterioration, uncured malignancies, left ventricular ejection fraction $\leq 45 \%$, estimated glomerular filtration rate $\leq 35 \mathrm{~mL} / \mathrm{min}$, STEMI, mitral regurgitation, and medication at discharge with clopidogrel and statins (Table 2 and Figure 4).

\section{Discussion}

Our study demonstrated that in an octogenarian patient cohort presenting with ACS from daily clinical practice, there was an association between PCI and reduced all-cause death over 5 years of follow-up.

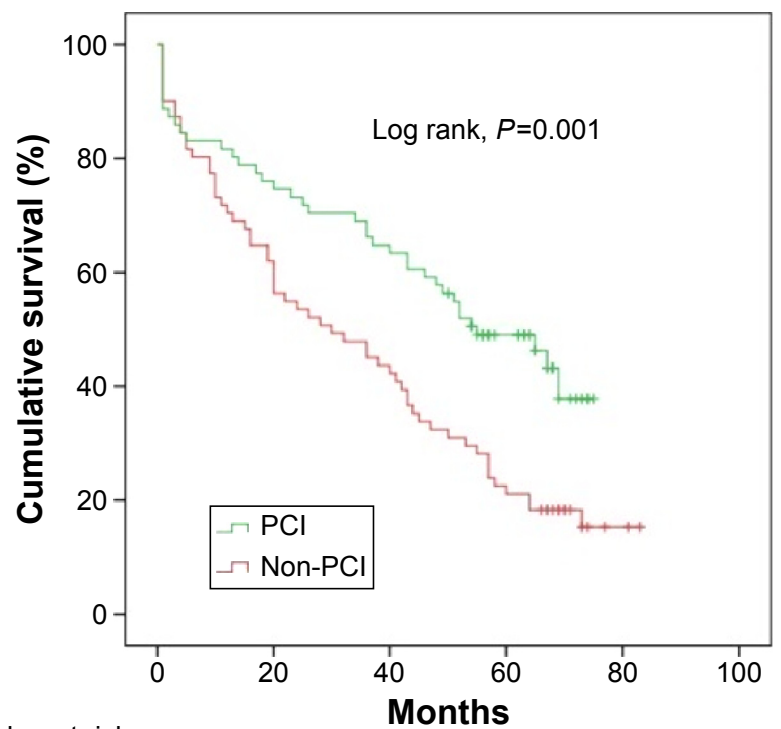

Number at risk:

$\begin{array}{lllll}\mathrm{PCl} & 71 & 53 & 45 & 35\end{array}$

$\begin{array}{lllll}\text { Non- } \mathrm{PCl} & 71 & 41 & 30 & 16\end{array}$

Figure 2 Kaplan-Meier survival curves for all-cause mortality of PCl-treated patients versus non- $\mathrm{PCl}$-treated patients in propensity-matched cohort. Abbreviation: $\mathrm{PCl}$, percutaneous coronary intervention. 


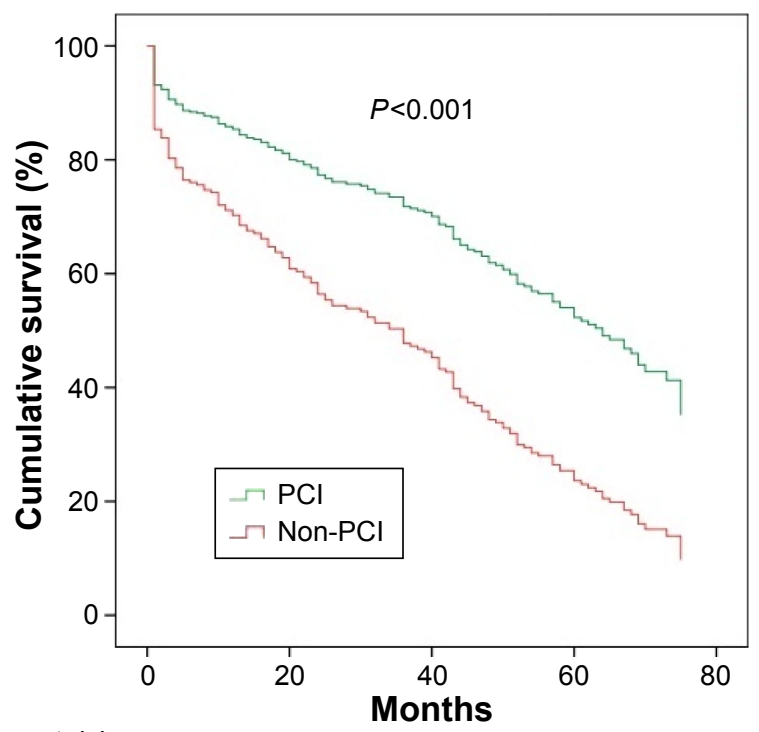

Number at risk:

$\begin{array}{lllll}\mathrm{PCl} & 182 & 146 & 127 & 95 \\ \text { Non-PCl } & 171 & 104 & 77 & 41\end{array}$

Figure 3 Cox regression survival curves for all-cause mortality of $\mathrm{PCl}$-treated patients versus non- $\mathrm{PCl}$-treated patients in the overall cohort (adjusted for confounders). Abbreviation: $\mathrm{PCl}$, percutaneous coronary intervention.

In overall cohort, there was a significant association between reduction in 5-year all-cause mortality and PCI. But due to differences in baseline characteristics between the two groups, a PS-based matching was performed. It is interesting to note that there was a similar association between all-cause mortality and PCI even in the PS-matched cohort. Consequently, PCI had a strong association with improved survival in our octogenarian cohort despite PS matching and adjustment with Cox proportional-hazard regression. Therefore, our study implies that among patients aged $\geq 80$ years, there is a potential prognostic benefit of PCI in patients presenting with ACS. In view of the fact that there are no prospective, randomized, controlled clinical trials available to compare head to head between PCI-treated and non-PCI-treated patients with ACS aged $\geq 80$ years, our results are hypothesis-generating and suggest that efficacy of PCI warrants further study in octogenarian ACS patients.

Our results are in line with recently published trials. Savonitto et al demonstrated that an early aggressive approach (coronary angiography, and when indicated, revascularization within 72 hours) resulted in a significant reduction in the primary endpoint (the composite of death, MI, disabling stroke, and repeated hospital stay for cardiovascular causes or severe bleeding within 1 year) in elderly patients with non-ST-segment elevation ACS. However, in this study, this positive outcome only occurs in those with

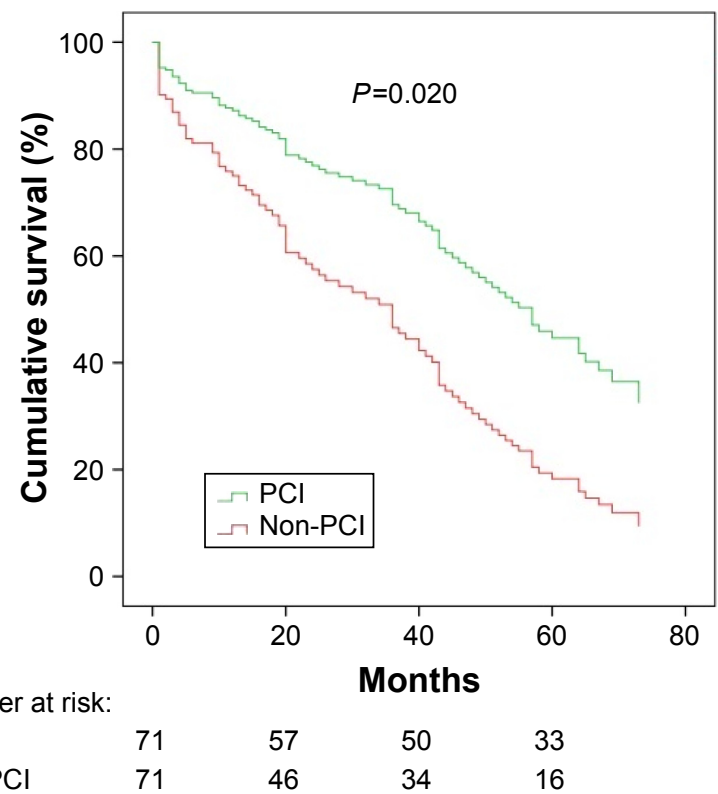

Figure 4 Cox regression survival curves for all-cause mortality of $\mathrm{PCl}$-treated patients versus non-PCl-treated patients in the matched cohort (adjusted for propensity score and confounders).

Abbreviation: $\mathrm{PCl}$, percutaneous coronary intervention.

elevated troponin on admission. ${ }^{17}$ In another multicenter study by Tegn et al elderly ACS patients who underwent an invasive strategy had a decrease in the primary composite endpoint (MI, need for urgent revascularization, stroke, and death) as compared with a conservative approach (not published yet). However, it is noteworthy to mention that there are several differences between above randomized studies and our retrospective study. For the first, in abovementioned two randomized studies, it is an early invasive approach that was studied, that is, PCI only when indicated, in comparison with conservative strategy. This implies that not all patients in the group assigned to invasive approach have undergone PCI. However, our study aimed to compare between PCI and non-PCI treatment. For the second, patient population included in above-mentioned randomized studies were either NSTEMI or stable ACS patients. Presumably, patients with unstable ACS or STEMI with strong clinical indication for acute PCI were excluded for randomization because of ethical reasons. However, in our study, because of nature of retrospective study, there were no pre-specified criteria for PCI rather than on the basis of clinical decision of responsible physician. Therefore, all patients with ACS including STEMI and cardiogenic shock were included.

\section{Study limitations/strengths}

In this study, secondary medical record data were used. Despite our efforts in catching up as much information as 
possible, there were still some data missing. Despite PS adjustment, we cannot rule out residual confounding from unmeasured variables. Therefore, our results need to be confirmed in a randomized controlled study in the future. Nevertheless, our data are encouraging and serve as the basis for generating hypothesis in future randomized trials. The strength of our study is that all patients were included from our daily clinical practice; therefore, the generalizability should be regarded as higher than those of randomized, controlled, clinical trials.

\section{Conclusion}

In an octogenarian patient cohort with ACS in daily clinical practice, PCI was associated with reduced all-cause death over 5 years of follow-up.

\section{Acknowledgment}

This study was kindly supported by the Swedish Heart-Lung Foundation.

\section{Disclosure}

The authors report no conflicts of interest in this work.

\section{References}

1. Hamm CW, Bassand JP, Agewall S, et al; ESC Committee for Practice Guidelines. ESC Guidelines for the management of acute coronary syndromes in patients presenting without persistent ST-segment elevation: The Task Force for the management of acute coronary syndromes (ACS) in patients presenting without persistent ST-segment elevation of the European Society of Cardiology (ESC). Eur Heart J. 2011;32(23): 2999-3054.

2. Van de Werf F, Bax J, Betriu A, Blomstrom-Lundqvist C, et al. ESC guidelines on management of acute myocardial infarction in patients presenting with persistent ST-segment elevation. Eur Heart J. 2008;29(23): 2909-2945.

3. Wijns W, Kolh P, Danchin N, Di Mario C, et al. Guidelines on myocardial revascularization. Eur Heart J. 2010;31(20):2501-2555.

4. Appleby CE, Ivanov J, Mackie K, et al. In-hospital outcomes of very elderly patients ( 85 years and older) undergoing percutaneous coronary intervention. CCI J. 2011;77(5):634-641.
5. de Boer SP, Westerhout CM, Simes RJ, et al; Primary Coronary Angioplasty Versus Thrombolysis-2 (PCAT-2) Trialists Collaborators Group. Mortality and morbidity reduction by primary percutaneous coronary intervention is independent of the patient's age. JACC. 2010;3(3): 324-331.

6. Shanmugasundaram M. Percutaneous coronary intervention in elderly patients: is it beneficial? Tex Heart Inst J. 2011;38(4):398-403.

7. Yan BP, Gurvitch R, Duffy SJ, et al. An evaluation of octogenarians undergoing percutaneous coronary intervention from the Melbourne Interventional Group registry. Catheter Cardiovasc Interv. 2007;70(7): 928-936.

8. Muñoz JC, Alonso JJ, Duran JM, et al. Coronary stent implantation in patients older than 75 years of age: clinical profile and initial and long-term (3 years) outcome. Am Heart J. 2002;143(4):620-626.

9. Varani E, Aquilina M, Balducelli M, Vecchi G, Frassineti V, Maresta A. Percutaneous coronary interventions in octogenarians: acute and 12 month results in a large single-centre experience. Catheter Cardiovasc Interv. 2009;73(4):449-454.

10. Hiew C, Williams T, Hatton R, et al. Influence of age on long-term outcome after emergent percutaneous coronary intervention for ST-elevation myocardial infarction. J Invasive Cardiol. 2010;22(6):273-277.

11. Klein LW. Percutaneous coronary intervention in the elderly patient (Part I of II). J Invasive Cardiol. 2006;18(6):286-295.

12. Thomas MP, Moscucci M, Smith DE, et al; Blue Cross Blue Shield of Michigan Cardiovascular Consortium. Outcome of contemporary percutaneous coronary intervention in the elderly and the very elderly. Clin Cardiol. 2011;34(9):549-554.

13. Schoenenberger AW, Radovanovic D, Stauffer JC, et al; Acute Myocardial Infarction in Switzerland Plus Investigators. Age-related differences in the use of guideline-recommended medical and interventional therapies for acute coronary syndromes: a cohort study. J Am Geriatr Soc. 2008;56(3):510-516.

14. Yan RT, Yan AT, Tan M, et al. Age-related differences in the management and outcome of patients with acute coronary syndromes. Am Heart J. 2006;151(2):352-359.

15. Avezum A, Makdisse M, Spencer F, et al; GRACE Investigators. Impact of age on management and outcome of acute coronary syndrome: observations from the Global Registry of Acute Coronary Events (GRACE). Am Heart J. 2005;149(1):67-73.

16. Shanmugasundaram M, Alpert JS. Acute coronary syndrome in the elderly. Cilin Cardiol. 2009;32(11):608-613.

17. Savonitto S, Cavallini C, Petronio AS, et al. Early aggressive versus initially conservative treatment in elderly patients with non-ST-segment elevation acute coronary syndrome: a randomized controlled trial. J Am Coll Cardiol Cardiovascular Intervention. 2012;5(9):906-916.
Clinical Interventions in Aging

\section{Publish your work in this journal}

Clinical Interventions in Aging is an international, peer-reviewed journal focusing on evidence-based reports on the value or lack thereof of treatments intended to prevent or delay the onset of maladaptive correlates of aging in human beings. This journal is indexed on PubMed Central, MedLine,

\section{Dovepress}

CAS, Scopus and the Elsevier Bibliographic databases. The manuscript management system is completely online and includes a very quick and fair peer-review system, which is all easy to use. Visit http://www.dovepress. com/testimonials.php to read real quotes from published authors. 